\title{
Del régimen de entidades controladas del exterior, características e implicaciones de su incorporación en el régimen tributario colombiano
}

\section{The Controlled Foreign Company, characteristics and implications of its incorporation in the Colombian Tax Regime}

\author{
JULIETA OSPINA GARCÍA ${ }^{1}$
}

\section{Resumen}

La Ley 1819 de 2016 incorporó el Libro VII al Estatuto Tributario, reglamentando las operaciones de las entidades controladas del exterior sin residencia en Colombia, un tema completamente nuevo para los contribuyentes y que tiene como objetivo el control de las rentas pasivas, que, además, no ha tenido una reglamentación adecuada ni una difusión para darle claridad al contribuyente sobre el cumplimiento de esta nueva legislación.

Este es un tema de poco conocimiento por parte de los contribuyentes obligados a cumplir con esta nueva reglamentación, y de escasa literatura que sirva como guía al contribuyente, por lo que un texto de esta magnitud permite darle al lector un panorama global integrado sobre los Artículos 882 al 893 del Estatuto Tributario, cuya primera aplicación deberá hacerse en las declaraciones del periodo fiscal $2017 \mathrm{y}$, seguramente, abordará dudas que a todos nos competen, tanto a contribuyentes como a asesores.

Palabras clave: Transparencia fiscal, ECE (Entidades controladas del exterior no residentes en Colombia), Acción 3 de BEPS, Control, Rentas cedulares, Dividendos,

${ }^{1}$ Contadora (1998), Especialista en Finanzas (2008), Especialista en Mercado de Capitales (2009) Universidad del Rosario, y Magíster en Tributación internacional, comercio exterior y aduanas (2018), Universidad Externado de Colombia. Tiene experiencia en el sector privado, actualmente se desempeña como consultora privada para diferentes sectores de la Economía con operaciones en Colombia y en el exterior. Correo-e: julieta_ospigarcia@outlook.es DOI: https://doi.org/10.18601/16926722.n13.05 
Compensación de impuestos pagados en el exterior, Convenios para evitar la doble imposición (CDI).

\section{Abstract}

Law 1819 of 2016 incorporated the VII Book to the Tax Statute, regulating the operations of foreign controlled entities without residence in Colombia, a completely new subject for taxpayers and whose propose is the control of passive income, which also hasn't had an adequate regulation or a diffusion to give clarity to the taxpayer on compliance with this new legislation. Issue of little knowledge by taxpayers forced to comply with this new regulation and with little literature to serve as a guide to the taxpayer, so a text of this magnitude can give the reader a comprehensive overview on articles 882 to 893 of the Tax Statute, whose first application should be made in the statements of the 2017 fiscal period and which will surely address doubts that all of us have to deal with both taxpayers and advisors

Keywords: Fiscal transparency, ECE (Foreign controlled entities not resident in Colombia), Action 3 of BEPS, Control, Structured income, Dividends, Compensation of taxes paid abroad, Agreements to avoid double taxation (CDI).

\section{Introducción}

Las más recientes reformas tributarias en Colombia han incorporado reglamentaciones que buscan cerrar las brechas generadas por las diferencias jurídicas en las legislaciones que gravan las rentas de los con- tribuyentes que se encuentran en distintas jurisdicciones. Es así como, bajo la potestad jurídica, algunos Estados gravan la renta con fundamento en el origen de estas, es decir, según la fuente, mientras otros gravan a los contribuyentes por la renta mundial, sin importar dónde se origine.

Colombia no ha sido ajena tampoco a la globalización de la economía y a los cambios que ella trae, como es la Economía digital. A la par de estos cambios, nuestra legislación ha encaminado sus esfuerzos a incorporar las mejores prácticas tributarias y la experiencia de otras jurisdicciones, así como las recomendaciones que la OCDE hace a través del plan de acción BEPS (Base Erosion and Profit Shifitng).

En consecuencia, la reforma tributaria de 2016 incorporó la reglamentación que rige las entidades controladas del exterior (CFC - Controlled Foreign Company), bajo el esquema de ECE (Entidades controladas del exterior no residentes en Colombia) la cual debe ser aplicada por un amplio número de contribuyentes, entre los que se encuentran personas naturales y jurídicas, por lo cual toma importancia ahondar en la legislación incorporada en el Libro VII del Régimen tributario colombiano, mediante la Reforma tributaria 1819 de 2016, y analizar el impacto que dicha legislación tendrá, no solo en los contribuyentes, sino también en la administración tributaria. En este contexto, el presente escrito tiene como objetivo analizar el impacto y la forma como se incorporó el proyecto BEPS a través de la reforma tributaria colombiana de 2016, con especial énfasis en la acción tres. 


\section{Origen de las normas de} transparencia fiscal internacional y las normas de CFC (Controlled Foreign Company)

Entre las acciones diseñadas está BEPS, y en ella, la número tres (3) denominada $\mathrm{CFC}$ (Controlled Foreign Corporations), cuyo objetivo es lograr que los ingresos pasivos obtenidos por las compañías controladas extranjeras sean declarados por la matriz en el mismo periodo en que dichos ingresos fueron generados en la CFC (en Colombia ECE).

Esta acción también busca proteger la tasa de tributación de tal forma que las normas CFC atacan primordialmente las operaciones que se realizan en jurisdicciones de baja o nula imposición o en regímenes preferenciales que tienen tasas inferiores a la de la Matriz.

El país que lideró la implementación de las normas CFC fue Estados Unidos después de la Segunda Guerra Mundial, con la Ley 88-780 en el año 1960. Desde ese momento, se han realizado diversas reformas que buscaron mejorar o ajustar las normas sobre las CFC; aunque algunas reformas han complementado la legislación en cuanto a su forma, otras han sido utilizadas para tratar de que los residentes normalicen los patrimonios que no han declarado y pagando tasas preferenciales. Esto último se vio en la reformas de 2004 y en la reciente reforma de 2017, Ley 115-97 (Tax Cuts and Job $A c t$ ), donde además se adicionan conceptos como el GILTI (Global Intangible Low-Taxed Income) y el QBAI (Qualified Business Asset Investment). Estados Unidos reglamentó hace más de 50 años las CFC y, además de que las reforma periódicamente, también las ha complementado con normas como la Ley de cumplimiento tributario de cuentas en el extranjero de sus residentes FACTA (Foreign Account Tax Compliance Act), vigente desde el 2013.

Otras jurisdicciones, como España, han implementado las normas CFC en el capítulo de transparencia fiscal, que deben aplicar las personas jurídicas como las personas físicas (término que hace referencia a las personas naturales). En general, la reglamentación de las normas CFC cubre la totalidad de las rentas pasivas, excluyendo actividades reales o rentas activas. Las normas no son aplicables a una entidad no residente en otro Estado miembro de la Unión Europea, de acuerdo con la Directiva 2009/65/CE del Parlamento Europeo; también se tiene una excepción con Luxemburgo para las entidades SIF (Specialized Investmet Funds) ${ }^{2}$, en cuanto a que si se demuestra que las operaciones se realizan con un sentido real, son sustanciales. En España se permite la compensación de impuestos pagados de periodos anteriores.

Las normas CFC en Perú están en vigencia desde el año 2013 y se encuentra reglamentado en el capítulo XIV del Régimen de transparencia fiscal internacional.

En cuanto a Chile, la figura está vigente desde el año 2016. Una característica que

${ }^{2}$ SIF: Fondos de inversión especializados, regulados por la Ley sobre SIF del 13 de febrero de 2017. 
distingue estas dos legislaciones es el hecho de que se tienen establecidos porcentajes para la presunción de ingresos y, en cuanto a las rentas pasivas, se hace referencia a la Unidad de Fomento (UF) en Chile, mientras que en el caso de Perú se hace referencia a la Unidad impositiva tributaria (UIT) las cuales, en ambos casos, son valores de referencia que se utilizan para determinar impuestos, infracciones, multas u otros aspectos de orden tributario.

En ambos países se estableció una excepción relativa a la satisfacción del impuesto. En la jurisdicción de la CFC equivalente al $35 \%$ o más del impuesto efectivo en Chile, mientras en Perú se tiene establecido un umbral del $75 \%$ de la tasa aplicable a los residentes. Es importante mencionar que en ambas legislaciones se excluyen las rentas originadas en actividades reales y, además, no existe la presunción por derecho ya que el contribuyente tiene el derecho de demostrar el origen de las rentas.

\section{La acción 3 del Plan BEPS (Erosión de la Base y Traslado Artificial de Utilidades)}

$\mathrm{Al}$ hacer un análisis de la reforma tributaria de 2016 en Colombia y la incorporación de las CFC (llamadas en dicha reforma ECE, Entidades controladas del exterior), se encuentra que no hubo una referencia introductoria a la acción 3 de BEPS en la exposición de motivos, pues esta acción es la que fundamenta el control sobre las entidades controladas del exterior no residentes en Colombia.

Más allá de lo anterior, es importante recordar que esta figura hace parte de un plan de acciones para tratar problemas relacionados con la erosión de la base fiscal y el traslado de beneficios.

La figura 2 muestra un resumen de las acciones que hacen parte del plan de Acción BEPS.

El plan de acción contra BEPS gira en torno a tres pilares fundamentales: A) que las normas de derecho interno de los países sean coherentes; B) que se analice e incorpore el criterio de actividad sustancial, y C) que los países cuenten con transparencia y seguridad jurídica en sus operaciones transfronterizas. Es por esto que la acción 3 de BEPS plantea una serie de consideraciones y recomendaciones acerca de los pilares fundamentales de las normas CFC, a fin de que al incorporarla a la legislación interna de cada país se haga efectiva, porque algunas jurisdicciones han establecido las normas CFC pero han sido incapaces de enfrentar las BEPS de una manera eficiente, teniendo que modificarla inclusive para lograr una mejora en la recaudación. Por ello, la OCDE no limita la incorporación de los pilares, de tal forma que puedan adaptarse a los objetivos políticos y fiscales de cada jurisdicción.

El informe final de acción 3 identifica las rentas pasivas que son usadas para la erosión de la base fiscal o el traslado de beneficios; así, con ella se pueden atacar algunas rentas que no cubren la normativa sobre precios de transferencia, de tal suerte que no necesariamente la acción 3 cumpliría los objetivos de la normativa de precios de transferencias.

Los pilares fundamentales de las normas de transparencia fiscal internacional (TFI) recomendados por la OCDE son: a) normas 
Figura 2. Plan de acción BEPS

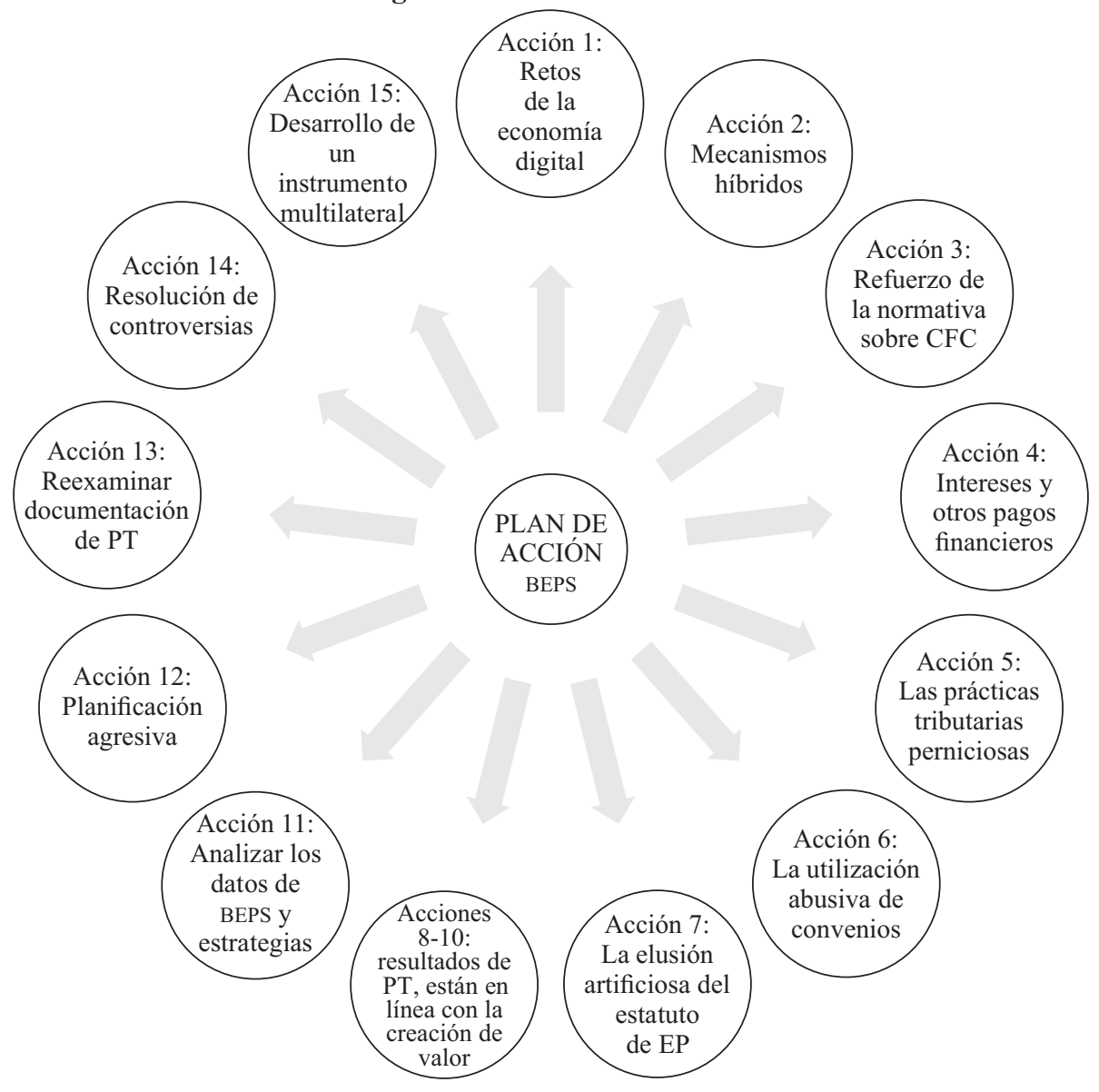

Fuente: Elaboración propia.

para definir una SEC (incluye las normas de control); b) umbrales mínimos y exenciones; c) definición de renta sujeta al régimen de TFI; d) normas para calcular la renta; e) normas para atribuir la renta; f) normas para prevenir o eliminar la doble imposición" (OCDE, 2016, p. 11).

En general, la recomendación de la OCDE es que en el diseño de las normas TFI se incluya un amplio grupo de entidades, no solo aquellas que per se puedan ser consideradas como CFC, sino también aquellas entidades transparentes como las sociedades de personas (partnerships) o establecimientos permanentes (EP) que, aunque tengan una operación real, puedan constituir un vehículo por obtener rentas que susciten riesgos BEPS.

También "se recomienda que se incorporen normas antihíbridos, con el fin de evitar que las entidades eludan las normas TFI" por tener un tratamiento jurídico diferente en otras jurisdicciones. 
En relación con las entidades transparentes, aunque sus rentas ya estén gravadas en cabeza del propietario, se debe verificar si la entidad obtiene un ingreso que pueda ser un riesgo BEPS o que no esté gravado en la jurisdicción de la Matriz por algún tipo de exención aplicable en dicha jurisdicción.

Así mismo, se evalúa si una $\mathrm{CFC}$ es dueña de una entidad transparente que pueda ser usada para trasladar beneficios que le permitan erosionar la base gravable (OCDE, 2016, p. 23).

En lo referido a los Establecimientos Permanentes (EP), la recomendación se centra en establecer si la entidad no residente tiene un EP en otra jurisdicción diferente de la matriz y para los casos en los que la jurisdicción de la matriz deja exenta la renta del EP.

En cuanto a la incorporación de las normas antihíbridos, deben ser analizadas especialmente en las operaciones intragrupo, donde el pagador y el beneficiario del pago tengan un tratamiento igual por pertenecer a un grupo económico, sin tener en cuenta que para el pagador el desembolso puede ser calificado por la jurisdicción de la matriz como un costo deducible de renta.

En lo referente al control, se recomienda la incorporación de un test jurídico, otro test de control económico, y en algunas jurisdicciones también se podría incorporar un test de facto, en la medida en que, en algunas ocasiones, los dos primeros pueden ser manipulados por los contribuyentes, especialmente cuando los tests deban hacerse al cierre fiscal y no se considere el control en cualquier día del periodo fiscal (como sucede en algunas jurisdicciones).

En lo que refiere a la participación, se recomienda que el porcentaje sea al menos de un $50 \%$, pues el nivel de control puede determinarse sumando los porcentajes de participación de partes residentes vinculadas o no vinculadas, o sumando la participación de todos los contribuyentes residentes que actúan de manera concertada, esto, independientemente de que el control sea directo o indirecto.

\section{Configuración legal del régimen de entidades controladas del exterior no residentes en Colombia}

\section{A. Incorporación del régimen $\mathrm{CFC}$, un nuevo paso en el camino para afrontar los desafios de la globalización}

Los primeros pasos que dio Colombia hacia la incorporación de las normas CFC los dio en la Ley 1739 de 2014, cuando se adicionó el Artículo 607 al Estatuto Tributario, estableciendo la declaración de los activos que se tuvieran en el exterior como una obligación que debían cumplir los contribuyentes del impuesto de renta y complementarios, y normalizar sus patrimonios en el exterior a una tarifa reducida.

La reglamentación del régimen de entidades controladas del exterior sin residencia en Colombia (ECE), es una consecuencia lógica de un proceso de mejoramiento fiscal en el que se viene trabajando hace mucho tiempo, aunque existen otras iniciativas que buscan los mismo: a) el intercambio automático de información entre las administraciones tributarias; b) las normas antielusión; c) la 
revelación del beneficiario efectivo, y d) revelación de los esquemas de planeación tributaria agresiva utilizada por los contribuyentes (planteado en el proyecto de reforma tributaria, sin éxito), las normas de abuso en materia tributaria, así como el mejoramiento de la normativa relacionada con precios de transferencia, muestran las intenciones del gobierno en atacar contundentemente la evasión en el corto plazo así como las practicas elusivas.

Finalmente, en la reforma tributaria de la Ley 1819 del 2016, mediante el Artículo 139 se incorporó el Libro séptimo al Estatuto Tributario y los Artículos 882 al 893, reglamentando toda la operación de las ECE (entidades controladas del exterior sin residencia fiscal en Colombia).

\section{B. Entidades controladas del exterior sin residencia en Colombia, según la Ley 1819 de 2016}

Según el Artículo 882 del ET, se debe cumplir con dos condiciones para encontrarse ante una ECE: una de ellas es que la entidad no sea "residente fiscal en Colombia", y además que se pueda establecer el control en los términos de algunas de las situaciones establecidas en el Artículo 260-1 del Estatuto Tributario en sus numerales i., ii., iv., y) v. del literal b del Numeral 1 o que exista vinculación económica en cualquiera de los supuestos del numeral 5 del citado artículo.

También incluye diferentes vehículos de inversión (parágrafo 3) como las opciones de compra de acciones o participaciones de la ECE, y no podían quedarse por fuera las
ECE que están ubicadas en jurisdicciones de baja o nula tributación o con un régimen impositivo preferencial en los términos del artículo 260-7 del Estatuto Tributario.

\section{Criterios de vinculación para efectos de determinar el control}

El primer análisis se enfoca en los criterios de vinculación por subordinación, tal y como lo establece el Artículo 882 del E.T. Numeral 1 , literal a y $b$, que remite a los criterios de vinculación el Artículo 260-1 del ET. De acuerdo con esta reglamentación, podemos concluir que se incorporan el control económico y el control jurídico, esto por las características del Artículo 260-1 del ET.

Es importante conocer que el Artículo 260-1 del E.T. es el que reglamenta las operaciones de precios de transferencias, situación ampliamente conocida por los grandes empresarios, pero totalmente nueva para otros contribuyentes, especialmente personas naturales, quienes, sin cumplir con el umbral previsto para el régimen de precios de transferencias (actualmente 100.000 UVT de patrimonio bruto o 61.000 UVT de ingresos brutos, así como lo estipulado en el Artículo 2 del decreto 3030 de 2013), deberán hacer estos análisis de vinculación para cumplir con lo estipulado en el Artículo 882 del Estatuto Tributario.

Finalmente, el parágrafo 3 del Artículo 882 del Estatuto Tributario establece que se considera que un residente colombiano tiene control sobre una ECE cuando esta última se encuentre ubicada, constituida o en operación en una jurisdicción de baja o nula imposición, así como si la ECE se encuentra sometida a un régimen tributario 
preferencial, en los términos del Artículo 260-7 del ET.

El Gobierno nacional no observó las recomendaciones de la OCDE, donde se indica que la reglamentación de precios de transferencia sea diferente a la de las ECE, ya que ambos persiguen objetivos diferentes, aunque complementarios, obligando a los contribuyentes a hacer un análisis de control y vinculación además de establecer si la entidad se encuentra ubicada en un régimen preferencial o de nula o baja imposición.

\section{Análisis de la condición de la no residencia de la EC}

Un segundo aspecto que contempla la legislación colombiana en el Artículo 882 del ET para clasificar una ECE es la calidad de NO residente, condición lógica teniendo en cuenta la naturaleza de este tipo de entidades; es importante determinar que en la legislación colombiana las sociedades o entidades son consideradas nacionales en cuanto cumplan con lo establecido en el Artículo 12-1 del Estatuto Tributario, es decir, cuando: "a) tengan la sede efectiva de negocios en el territorio colombiano, b) tengan su domicilio en Colombia y c) cuando han sido constituidas bajo la legislación nacional". En esas condiciones, cuando una entidad cumpla con los criterios de vinculación del numeral 1 literal a y/o b del Artículo 882 del ET, se deberá establecer si en efecto cumple con alguno de los requisitos de residencia para calificarla como ECE, o si siendo una sociedad extranjera tiene su sede efectiva de administración en Colombia, caso en el cual deberá ser tratada como una entidad residente para todos los efectos fiscales y no podría ser clasificada como ECE. Ahora bien, el parágrafo 6 del Artículo 12-1 del E.T. aclara que no se entenderá que existe sede efectiva de administración cuando las entidades extranjeras reciban ingresos del exterior iguales o superiores al $80 \%$ de los ingresos totales de la compañía, sin tener en cuenta las rentas pasivas.

\section{Análisis sobre los vehículos de inversión que son considerados como entidades controladas del exterior sin residencia en Colombia (ECE)}

Los parágrafos 1 y 3 del Artículo 882 del E.T. enuncian que serán considerados como ECE los siguientes vehículos de inversión: “a) sociedades, b) patrimonios autónomos, c) trusts ${ }^{3}$, d) fondos de inversión colectiva, e) otros negocios fiduciarios, y las fundaciones de interés privado"'; también, establece que la tenencia de opciones de compra sobre acciones o participaciones también será considerada como una tenencia directa para efectos de control.

Para el caso de los derechos fiduciarios y los trusts que no tienen una definición exacta en el ET, pero que según el Artículo 37 de la Ley 1739 de 2014 se asimilan a los derechos fiduciarios, se establece en el Artículo 102

\footnotetext{
${ }^{3}$ Trust: es una relación jurídica en la que la persona denominada settlor cede una propiedad a otra de su confianza (trustee) para que la administre en beneficio de terceros y/o con una finalidad específica.

${ }^{4}$ Fundaciones de interés privado (FIP): es una persona jurídica hibrida entre un fideicomiso y una compañía, que se constituye cuando el fundador traspasa sus bienes para que sean administrados por el consejo fundacional, en beneficio de sus beneficiarios.
} 
del ET en el inciso 2 "el principio de transparencia aplicable a los derechos fiduciarios, mediante el cual los beneficiarios del derecho, deberán incluir en sus declaraciones de renta los ingresos, costos y gastos devengados con cargo al patrimonio autónomo, en el mismo año gravable en que se devenguen a favor o en contra del patrimonio autónomo en las mismas condiciones tributarias en estas se incluyen la fuente, naturaleza, deducibilidad y concepto que tendrían si las actividades fueran realizadas directamente por el beneficiario, haciendo una referencia a que las personas naturales observarán las reglas de realización establecidas en el Art. 27 del ET". Según este artículo, los contratos fiduciarios, así como los trusts, serán entidades transparentes.

Ahora bien, según lo mencionado, los contribuyentes del impuesto a la renta deberán reportar los ingresos, costos y gastos con cargo al encargo fiduciario en el mismo periodo de realización de estos, esto llevaría a dudar al contribuyente entre aplicar el régimen de transparencia del Artículo 102 del ET, o la normativa de ECE para declarar la renta neta que se genere en la operación fiduciaria o del trust.

Este no es solo un problema procedimental sino que también hace referencia a la compensación de las pérdidas que puedan presentarse en fiducia o trust, las cuales podrán ser compensadas en los 12 periodos gravables siguientes por el contribuyente, ya que no tienen restricción según lo establece el Artículo 147 del ET, mientras bajo la norma CFC las pérdidas no pueden ser compensadas con las utilidades del contribuyente, ni en el periodo corriente ni en periodos posterio- res, podría entonces considerarse que si el contribuyente incorpora estas rentas según lo establecido en el Artículo 102, deberá demostrar ante la administración tributaria que los recursos son obtenidos en desarrollo de una actividad sustancial y no por una renta pasiva.

\section{4. ¿Cuándo es aplicable el régimen de acuerdo con el control y subordinación?}

Según lo incorporado en el Artículo 883 de Estatuto Tributario, cuando se identifique que la ECE es controlada por residentes colombianos, estarán obligados a cumplir con esta obligación los contribuyentes del impuesto de renta y complementarios que ostenten una participación directa o indirecta igual o superior al $10 \%$ del capital o de los resultados de la ECE. Para esto, deberá reconocerse en las declaraciones de impuestos la renta pasiva que será el resultado de tomar los ingresos de la ECE y descontar los costos y deducciones asociados a esa renta, en la proporción de la participación que se tenga, es decir, quienes tengan menos del $10 \%$ no deberán cumplir con esta obligación.

Bravo, J. (2017, p. 37) hace un análisis del Artículo 883, estableciendo la posibilidad de que si se da aplicación al Artículo 28 del Código Civil, "significado de las palabras", que dice que las palabras serán interpretadas en su sentido natural y obvio, mientras el legislador no las haya definido expresamente, caso en el cual se aplicará la definición del legislador; es posible que los únicos sujetos pasivos obligados a cumplir con las normas sobre ECE sean las personas naturales, toda vez que el Artículo 10 del Estatuto Tributario establece que: 
[Art.10. Residencia para efectos tributarios: se consideran residentes en Colombia para efectos tributarios las personas naturales que cumplan con cualquiera de las siguientes condiciones...]

Lo anterior es complementado por el autor con el Artículo 30 del Código Civil, que indica que el contexto de la ley permite establecer el alcance de la misma, es decir, que a pesar de que el artículo no haga referencia explícita a que también son sujetos obligados las sociedades y solo haya sido establecida para "los residentes", por el contexto y la redacción de la misma se deduce que tanto personas naturales como jurídicas están obligadas a la aplicación del régimen. No obstante lo anterior, es posible que esta misma interpretación sea para otras personas un motivo de incumplimiento de la norma de ECE, es por esto que sería recomendable que el Artículo 883 del ET indicara que cuando se determine que la entidad es controlada por "Las personas naturales residentes en el país y las sociedades y entidades nacionales,...", tal y como lo establece el Artículo 254 del E.T., pero en referencia a los impuestos pagados en el exterior.

\section{Descripción de las rentas pasivas}

El Artículo 884 del ET reglamentó las rentas que pueden ser consideradas ingresos pasivos, entendiéndose estos como los ingresos que se generen sin que se involucre una actividad sustancial o, como algunos las denominan, "rentas sin necesidad de trabajar". Para el efecto, la reglamentación colombiana considera los siguientes ingresos como pasivos.

Nótese como el legislador solo consideró la sustancia en las operaciones para las ren- tas por dividendos, desconociendo no solo las recomendaciones de la OCDE sino también pronunciamientos de la Corte Constitucional, lo cual es mencionado por Bravo González, J. (2017, p. 26), con referencia a la sustancia de las operaciones, indicando que "las operaciones deben ser observadas dándole prelación a la sustancia sobre la forma y que el principio de prevalencia del derecho sustancial no puede ser ajeno al sistema tributario" (MP. Cifuentes Muñoz, E., en Sentencia C15-Corte Constitucional, 1993).

Dado lo anterior y los pronunciamientos de la Corte, el legislador debió conservar el espíritu de la norma CFC, y establecer algunos de los tests que la OCDE recomienda para evaluar la capacidad real de la ECE en la generación de esas rentas, así como otros aspectos que demuestren que los ingresos tienen su origen en actividades reales, como puede ser la asignación porcentual o umbrales para determinar cuáles rentas son activas y cuáles pasivas, excluyendo las activas de la obligación derivada de la normativa ECE.

Otros ingresos que no fueron considerados como rentas pasivas fueron los relacionados con seguros. El riesgo de este tipo de rentas es que los ingresos de la ECE pueden ser trasladados a una jurisdicción diferente y, según la OCDE, son tres (3) supuestos los que hacen más peligrosos este tipo de operaciones: a) cuando la ECE está sobrecapitalizada en relación con las entidades del negocio comparables; b) cuando el tomador del seguro, el asegurado, el beneficiario o los riesgos están ubicados en una jurisdicción diferente a la de la ECE, y c) cuando la renta de seguros deriva de contratos con partes vinculadas, en especial cuando la vinculada recibe algún 
Tabla 1. Descripción de las rentas pasivas

\begin{tabular}{|c|c|}
\hline Tipo de ingreso pasivo & Excepción \\
\hline $\begin{array}{l}\text { Dividendos, retiros o repar- } \\
\text { tos o realización de utilidades } \\
\text { provenientes de participación } \\
\text { en sociedades o vehículos de } \\
\text { inversión. }\end{array}$ & $\begin{array}{l}\text { a. Que las utilidades provengan de rentas activas de la ECE, sus filiales, subordinadas, } \\
\text { o EP. } \\
\text { b. Que las utilidades se generen en la misma jurisdicción donde tenga residencia fiscal } \\
\text { la ECE, sus filiales, subordinadas, o EP. } \\
\text { c. Que exista control indirecto por parte de un residente colombiano en las filiales, } \\
\text { subordinadas o EP, de la ECE que genera las utilidades. } \\
\text { d. Cuando se hace referencia a actividades reales, significa dichas utilidades o repartos } \\
\text { se derivan en un porcentaje igual o superior al } 80 \% \text { de ingresos que no son conside- } \\
\text { rados rentas pasivas. } \\
\text { e. Que los dividendos, repartos o retiros, al haber sido distribuidos a los residentes } \\
\text { colombianos, hubieran estado exentos de tributación en Colombia, en virtud de un } \\
\text { convenio para evitar la doble imposición. }\end{array}$ \\
\hline $\begin{array}{l}\text { Intereses o rendimientos fi- } \\
\text { nancieros }\end{array}$ & $\begin{array}{l}\text { a. Aquellas que sean obtenidas por una ECE controlada por una sociedad nacional, que } \\
\text { a su vez esté sometida a inspección y vigilancia de la superintendencia Financiera de } \\
\text { Colombia. } \\
\text { b. Aquellas generadas por una institución financiera del exterior que no esté domici- } \\
\text { liada, constituida o localizada en una jurisdicción de baja o nula tributación, que no } \\
\text { haga intercambio efectivo automático de información con Colombia. }\end{array}$ \\
\hline $\begin{array}{l}\text { Ingresos de activos intangi- } \\
\text { bles }\end{array}$ & No tiene excepción \\
\hline $\begin{array}{l}\text { Ingresos generados por la ena- } \\
\text { jenación de activos que gene- } \\
\text { ren rentas pasivas }\end{array}$ & No tiene excepción. \\
\hline $\begin{array}{l}\text { Ingresos provenientes de bie- } \\
\text { nes inmuebles (arrendamiento } \\
\text { o enajenación). }\end{array}$ & No tiene excepción. \\
\hline $\begin{array}{l}\text { Ingresos provenientes de la } \\
\text { compra y venta de bienes cor- } \\
\text { porales }\end{array}$ & $\begin{array}{l}\text { Para que sean rentas pasivas, deben cumplir totalmente con lo siguiente: } \\
\text { a. Que sean adquiridos de, para o en nombre de una parte relacionada. } \\
\text { b. Que sean producidos, manufacturados, construidos, cultivados o extraídos en una } \\
\text { jurisdicción distinta a la residencia o ubicación de la ECE. } \\
\text { c. Que su consumo o disposición se realice en una jurisdicción distinta a la de la ECE. } \\
\text { d. Lo anterior significa que, si no cumple con los tres puntos anteriores, no se consi- } \\
\text { derarían rentas pasivas. }\end{array}$ \\
\hline $\begin{array}{l}\text { Ingresos provenientes de la } \\
\text { prestación de servicios téc- } \\
\text { nicos, de asistencia técnica, } \\
\text { administrativos, ingeniería, } \\
\text { arquitectura, científicos, ca- } \\
\text { lificados, industriales y co- } \\
\text { merciales. }\end{array}$ & $\begin{array}{l}\text { Para que sean considerados rentas pasivas de la ECE, este tipo de ingresos deberán: } \\
\text { a. Ser generados para o en nombre de partes relacionadas en una jurisdicción distinta } \\
\text { a la de residencia o ubicación de la ECE. } \\
\text { Los demás ingresos que no cumplan con el requisito anterior no podrán ser conside- } \\
\text { rados rentas pasivas. }\end{array}$ \\
\hline
\end{tabular}

Fuente: Elaboración propia.

tipo de deducción en sus rentas por el pago de la prima de seguro.

En cuanto a las rentas provenientes del arrendamiento o cesión temporal de bienes inmuebles, nuestra legislación no hizo ninguna excepción desconociendo así actividades reales que se realizan en este tipo de negocios, mientras que en el caso de Perú y Chile sí existe la excepción con referencia 
a estas rentas. Es importante aclarar que la renta de bienes inmuebles no está considerada con renta pasiva según el reporte final de la OCDE de 2015.

Según (Zarama, F. y Zarama, C., 2017) otro aspecto que genera dudas en el cálculo del control indirecto de una compañía es el cómputo que fue presentado como ejemplo en la exposición de motivos de la reforma tributaria, ya que cuando hay una cadena de control que a todas luces es directo desde el residente colombiano hasta la vinculada a una subsidiaria, el cálculo porcentual de la participación es por multiplicación, y a pesar de que la vinculada a la subsidiaria genere dividendos, si el umbral es inferior al 50\% establecido en la reglamentación, dichas rentas deberán ser declaradas como rentas pasivas, perdiendo la excepción que trae la norma cuando la vinculada de la subsidiaria se encuentre en una jurisdicción de nula o baja imposición. Ahora bien, es importante aclarar que no existe ninguna reglamentación del gobierno que indique la fórmula a aplicar para establecer el control directo, y esta conclusión solo se genera por el análisis prestado por los autores de la exposición de motivos.

Hecho el análisis anterior, se puede concluir que la legislación trató de incorporar análisis mecánicos y no detallados de operaciones para catalogar las rentas, y que aún tenemos aspectos que pueden ser mejorados en el futuro, en especial en lo referente a catalogar rentas como pasivas cuando pueden tener su origen en actividades reales, lo que permite especular que a futuro podríamos tener reglamentación complementaria a las normas de las rentas pasivas de las ECE, ya que como se ha presentado en otras jurisdicciones es posible que los contribuyentes decidan presentar ante los tribunales casos de actividades reales, para que no se les aplique el régimen ECE, tal y como pasó en el famoso caso de Cadbury Schweppes.

\section{Presunción de derecho de las ECE}

Finalmente, y como uno de los aspectos más criticables a la legislación es con referencia a las rentas pasivas, es importante analizar el Artículo 885 del Estatuto Tributario, que estableció que se presume de pleno derecho que cuando los ingresos pasivos de la ECE representen un $80 \%$ o más de los ingresos totales de la ECE, la totalidad de ingresos, costos y gastos darán origen a rentas pasivas; esto quiere decir que no admite prueba en contrario. Sin duda, lo que buscó esta norma fue facilitar el sistema fiscalizador de la administración tributaria, desconociendo además que el artículo que reglamenta la ECE en el Estatuto Tributario está enlazado con la normatividad de precios de transferencia, donde el contribuyente tiene que probar no solo la existencia real de la operación, sino que la misma se hace a precios de mercado, lo que debería ser suficiente para la administración como prueba en contrario a la presunción de derecho.

\section{Umbrales mínimos/exenciones}

La legislación colombiana no consideró las recomendaciones que hace la OCDE con referencia a la satisfacción de los impuestos en el exterior, o de al menos un porcentaje del impuesto que tendría que pagar la matriz residente para excluir las rentas pasivas de la obligatoriedad de cumplimiento de esta reglamentación. Dichas exenciones podrían ser complementadas con normas antidiferimiento o normas antiabuso, parecidas a las 
aplicadas en Estados Unidos, Chile y Perú; esto bajaría el impacto de la fiscalización a la administración tributaria.

\section{Normas para el cómputo y atribución de los ingresos de la ECE}

Desde el Artículo 886 al 891 del Estatuto Tributario está consignada toda la normativa que rige el reconocimiento de los ingresos, costos y gastos de las rentas pasivas, siguiendo el principio de reconocimiento igual a la normativa vigente para los ingresos de fuente nacional. Finalmente, la renta que debe incorporarse en el denuncio rentístico es la renta neta resultante de descontar de la sumatoria de los ingresos pasivos de la ECE el total de costos y gastos en que se incurrió para la generación de dichas rentas en el mismo periodo en el que fueron asignadas las ECE. También, la normativa indica que cuando las rentas pasivas sean inferiores a cero, no serán objeto de declaración por parte del contribuyente que controla, limitando así la compensación de pérdidas de la ECE, muy ajustado a la recomendación de la OCDE; el reconocimiento se hará en proporción al porcentaje de participación. Llama la atención que la reglamentación hace referencia a los artículos sobre reconocimiento de ingresos, deducciones y gastos para las rentas ordinarias, y esto me hace preguntar si el contribuyente deberá observar las reglas de realización de los costos de los Artículos 58 y 59 del E.T., muy propios de nuestra legislación, y en el caso de no cumplirlas, entonces, existirán costos que no podrá descontar de las rentas pasivas para determinar la renta neta a declarar.

Ahora bien, en cuanto a la compensación de los impuestos pagados en la jurisdicción por la renta de la ECE, el Artículo 892 sobre el crédito fiscal en Colombia por los impuestos pagados por la ECE en el exterior, indica que debe seguirse la regla del Artículo 254 del ET, el cual tiene características específicas en aspectos como: i) el procedimiento mediante una fórmula para determinar el valor del impuesto que puede ser descontado; en esta regla aún se tienen contemplados impuestos inexistentes, como el de la tarifa del impuesto a la equidad CREE y la sobretasa al CREE; ii) los impuestos pagados en el exterior pueden ser descontados hasta en los cuatro años siguientes a su pago; iii) además, el Artículo 254 del ET establece que para que puedan ser descontados estos impuestos, el contribuyente deberá tener control directo o indirecto, que la inversión que genere el descuento sea un activo fijo para el contribuyente y haberlo poseído por al menos dos años. Esto último claramente puede convertirse en un inconveniente al momento de justificar el descuento ante la administración tributaria, toda vez que es posible que el contribuyente tenga registrada la inversión de acuerdo con la liquidez esperada y no necesariamente registrada como activo fijo.

\section{Distribución de beneficios de una ECE previamente sometidos al régimen ECE}

Ahora bien, muchos contribuyentes pensarán que sus rentas serán doblemente gravadas en el momento de la incorporación por la aplicación del régimen de ECE y cuando sean efectivamente distribuidas por la ECE. Para esto, el Estatuto Tributario establece en el Artículo 893 que las rentas que previamente hayan sido sometidas a tributación por el régimen de ECE deberán ser tratadas como ingresos no constitutivos de renta ni ganancia ocasional, condicionando dicho tratamiento a las normas establecidas en el literal b) numeral 2 del Artículo 49 del Estatu- 
to Tributario. Sin embargo, por la redacción del numeral 2 del literal b del mencionado artículo, este no puede tomarse de forma literal e independiente, debiendo analizar el contexto del mismo en su totalidad. Ahora bien, el Artículo 49 establece que el contribuyente debe determinar el valor máximo susceptible de ser distribuido a título de ingreso no constitutivo de renta ni de ganancia ocasional, debiendo entonces tomar la renta líquida gravable adicionada con la renta por ganancias ocasionales, descontando los impuestos de renta y de ganancia ocasional, así como los impuestos pagados en el exterior según los literales a), b) y c) del inciso segundo en el Artículo 254 del ET, esto puede llevar a que el contribuyente pague un doble impuesto sobre una proporción del ingreso que tras la aplicación de esta regla no pueda ser considerado "ingreso no constitutivo de renta ni ganancia ocasional"; hubiera sido más conveniente que este ingreso fuera clasificado como una renta exenta sin sujetarla a ningún otro artículo del E.T.

\section{Interacción con los convenios para evitar la doble imposición}

Aunque existen algunos autores que consideran que se debe establecer claramente el manejo de los CDI con las normas CFC (ECE en Colombia), es claro que los acuerdos CDI están jurídicamente sobre la legislación nacional previa y posterior a la firma del acuerdo. Ahora bien, es importante resaltar que los mismos tratados contemplan normas para el abuso de tratados y la facultad que tiene la administración tributaria de reconfigurar las rentas amparadas por el tratado y desconocer los beneficios a los que se hayan sometido. Según Cabrera Cabrera, O. (2017, pp. 240243), los tratados que están bajo el Modelo de la OCDE no tendrán un riesgo de doble im- posición debido a las reglas de distribución de las rentas obtenidas por los países firmantes del convenio. Sin embargo, los tratados firmados bajo el modelo de la ONU (Chile, México y Portugal) podrán ser gravados bajo la norma del tratado que permite grabar en la fuente los ingresos catalogados en "otras rentas", que son los ingresos que no puedan ser clasificados en ninguna de las cláusulas del convenio y que por esa razón deban ser tratados como "otras rentas".

\section{Algunos aspectos problemáticos de la aplicación del régimen ECE en Colombia}

Unos de los principales desafíos que deben sortear los contribuyentes es determinar si reconocen o no el ingreso de la ECE en sus declaraciones privadas, lo anterior por el artículo incorporado sobre la realización de los ingresos, así:

Artículo 886: Los ingresos pasivos obtenidos por la ECE, se entienden realizados en cabeza de los residentes fiscales colombianos contribuyentes del impuesto de renta y complementarios que directa o indirectamente controlen la ECE, en el año o periodo gravable en que la ECE los realizó, en proporción a su participación en el capital de la ECE o en los resultados de esta última, según sea el caso, de acuerdo con los artículos 27, 28 y 29 del ET.

El Artículo 27 del Estatuto Tributario hace referencia a la realización del ingreso para los no obligados a llevar contabilidad, y el 28 para los obligados a llevar contabilidad. Ahora bien al respecto, tendrá el contribuyente que determinar si la ECE estaría obligada a llevar contabilidad en la jurisdicción donde esté ubicada, o si la remisión de los 
Artículos 27 y 28 hace referencia a los contribuyentes residentes obligados a llevar contabilidad y que deben declarar las rentas pasivas. Este es un interrogante que algunos autores, como Bravo González, J. (2017, p. 57), han considerado que hace que el artículo sea aplicable para la ECE y que sea para esta última para la que se deba determinar la aplicación del Artículo 27 o el 28.

\section{A. Incorporación en las rentas cedulares para las personas naturales}

Otro de los cambios introducidos por la Ley 1819 de 2016 es la reglamentación de las rentas para las personas naturales, cambiando de categorizar las rentas por la persona a categorizarlas al origen de las rentas como rentas cedulares.

Una de las primeras inquietudes que surgen en la incorporación de la renta de la ECE para las personas naturales es la que resulta de la aplicación del Artículo 890 del ET, denominado "Renta líquida gravable", y la inquietud surge porque, precisamente para el caso de las personas naturales, el formulario presenta dos alternativas: i) la renta líquida ordinaria y ii) las rentas gravables. En el primer caso, se puede depurar la renta incorporando y declarando ingresos, costos y gastos, mientras en el segundo caso solo se declararía el resultado de descontar a los ingresos los costos y gastos. En mi interpretación y de acuerdo con lo reglamentado en el decreto 2250 de 2017, los ingresos pasivos deberán ser declarados en cada cédula de acuerdo con el origen de la renta, que serían las de dividendos, la cédula de rentas de capital y la cédula residual de rentas no laborales.
La nueva determinación de las rentas de las personas naturales, según el Artículo 330 del ET, se clasifica en: a) rentas de trabajo; b) pensiones; c) rentas de capital; d) rentas no laborales, y e) dividendos y participaciones.

La incorporación del sistema cedular no solo afecta la clasificación que debe hacer el contribuyente de los ingresos obtenidos en el periodo gravable, sino también las tablas de tarifas aplicables, las cuales, ahora, después de la reforma tributaria, fueron agrupadas en 3 categorías: para las rentas laborales y las rentas de pensiones, será aplicable la tarifa del numeral 1 del Artículo 241 del Estatuto Tributario; a las rentas de capital y las rentas no laborales les será aplicable la tarifa del numeral 2 del Artículo 241 y, finalmente, a las rentas obtenidas por dividendos y participaciones, se les aplicará la tarifa establecida en el numeral 3 del Artículo 241.

Las pérdidas de las rentas líquidas cedulares no se sumarán para efectos de determinar la renta líquida gravable del periodo, y finalmente se incorpora el Artículo 333, donde se determina la base de la renta presuntiva para el cálculo de la renta por diferencia patrimonial.

Uno de los inconvenientes que pueden surgir, además de calificar las rentas en la cédula adecuada, es el hecho de que todas las rentas tienen un límite a las deducciones; además, el contribuyente deberá realizar un test propio que le permita garantizar aspectos como el control, la ubicación de la ECE, el origen de los ingresos, calificarlos como dividendos o como otro tipo de ingresos $y$, finalmente, verificar que la entidad que le genera las rentas pasivas no está ubicada en 
una jurisdicción de nula o baja tributación o que no intercambia efectivamente información con Colombia.

No son pocas las verificaciones que un contribuyente debería asegurar antes de incorporar esas rentas en su denuncio privado y aunque no se reglamentaron sanciones específicas para la omisión de rentas pasivas, sí existen otras sanciones aplicables, como son inexactitud en la declaración privada y la omisión de activos en el exterior.

\section{B. Rentas de dividendos}

Otro aspecto que genera controversia en su aplicación es como quedó redactado el Artículo 342 de la Reforma tributaria estructural Ley 1819 de 2016, donde se establece que son ingresos de las rentas de dividendos y participaciones los recibidos y constituyen renta gravable en cabeza de los socios (accionistas, comuneros, asociados, suscriptores y similares) que sean personas naturales residentes, provenientes de sociedades y entidades nacionales y entidades extranjeras.

Para determinar la renta líquida gravable, según el Artículo 343 del ET, se deberá dividir estas rentas en dos subcédulas: a) por los dividendos y participaciones provenientes de la distribución según el Artículo 49 numeral 3 del ET, y b) los que hayan sido distribuidos según el numeral 2 del Artículo 49 del ET y con los dividendos y participaciones provenientes de entidades extranjeras, al que se le aplicará la tarifa establecida en el inciso 2 del Artículo 242 del ET.

En lo que se refiere a las rentas de dividendos de las personas naturales no obligadas a llevar contabilidad, la remisión que hace el
Artículo 886 del ET a los Artículos 27, 28 y 29 sobre realización de los ingresos pasivos de la ECE desvirtúan el espíritu de las normas CFC, ya que la realización de los ingresos, según el Artículo 27 del ET, establece que las rentas de dividendos serán gravadas en el año o periodo en que sean realizadas, y que los "ingresos recibidos por anticipado" no se entenderán realizados hasta que no sean recibidos efectivamente por los contribuyentes, ya sea en dinero o en especie (Estatuto Tributario 2017), posición también presentada por Bravo González, J. (2017, p. 57), quien considera que, en el caso de los dividendos tanto para los obligados como los no obligados a llevar contabilidad, según los Artículos 27 y 28 se entenderán realizados "por los respectivos socios, accionistas ... cuando les hayan sido abonados en cuenta en calidad de exigibles". Ahora bien, en cuanto a los dividendos de los obligados a llevar contabilidad, están regidos por el Artículo 28 del E.T., y este a su vez remite al Artículo 27 numeral 1. En este caso, y teniendo en cuenta que la reglamentación de las ECE no contempló la documentación que debía conservar el contribuyente sobre las rentas pasivas, me hace preguntarme, ¿cómo puede la administración comprobar que una entidad del extranjero, donde el contribuyente tiene control y debería declarar los ingresos, no lo hace porque no le han sido "abonados los dividendos en calidad de exigibles"? Y en el caso de los no obligados a llevar contabilidad, dejar la opción del reconocimiento fiscal al momento del pago efectivo del dividendo o de que sea decretado, permite que los contribuyentes difieran el pago del impuesto a cargo, situación que precisamente quiere atacar la acción 3 de BEPS, que es el diferimiento del impuesto a cargo. 
Es importante aclarar que, para los obligados a llevar contabilidad, los otros ingresos deben ser reconocidos de acuerdo con el principio del devengo contable, según lo establece el Artículo 28, y salvo las excepciones en el mismo artículo, los otros ingresos deberán ser incorporados en las declaraciones de los contribuyentes que controlen la ECE.

Las rentas por dividendos de las personas naturales están consideradas en 3 artículos del Estatuto Tributario, el 27, el 28 y el 338, y para los tres el concepto es la recepción efectiva de los mismos para que surja la obligación de la declaración por parte del contribuyente, y las rentas de dividendos de la ECE remiten a los Artículos 27 y 28 para el reconocimiento fiscal del ingreso.

Aunque se esperaba una reglamentación de las rentas cedulares de las personas naturales y las ECE, solamente el Decreto 2250 del 29 de diciembre de 2017 estableció en el Artículo 1.2.1.20.8 que las rentas de la ECE deben ser declaradas en la correspondiente cédula de acuerdo con el origen de la renta. Ahora bien, el término "origen" puede interpretarse en dos vías: una que puede hacer referencia a que el origen es la ECE, o sea, la entidad, y la otra interpretación puede darse a la actividad que originó la renta pasiva, aplicando las normas de realización de ingresos, costos y gastos reglamentados en el Estatuto Tributario. En mi criterio, el decreto fue deficiente porque ante la novedad de la legislación, lo que se esperaba era una reglamentación que guiara al contribuyente en la aplicación del régimen de la ECE, como puede verse en la reglamentación de Perú, que explícitamente le indica al contribuyente la forma en que debe determinar la renta, en que cédula declararla y la tarifa aplicable.

Antes de incorporar las rentas por dividendos, los contribuyentes deberán verificar que las rentas que obtenga la ECE tengan origen en una actividad económica real, y si las rentas se generan por subordinadas, filiales o EP, que estas estén ubicadas en la jurisdicción de la ECE, y además debe verificar que exista control indirecto por uno o varios residentes colombianos en las filiales, subordinadas o EP de la ECE, además de que en sus registros contables sean reconocidas como inversiones y sean poseídas por más de dos años.

\section{Rentas originadas en actividades inmobiliarias}

Los ingresos provenientes de las actividades inmobiliarias que se obtengan a través de ECE, que deban ser declaradas por la persona natural, deberán ser incorporados en la cédula de rentas de capital con las mismas características y tarifas enunciadas anteriormente. No obstante, surgen dudas cuando los ingresos por actividades inmobiliarias sean desarrolladas por un fondo inmobiliario, que en esencia es una cartera colectiva donde el principal objetivo es la obtención de rentas para los inversionistas a través de la explotación comercial de un portafolio de activos inmobiliarios; la persona natural deberá declarar estas rentas en la cédula de dividendos, con las implicaciones económicas que ello deriva. Una dificultad que puede surgir en la aplicación de esta norma es que, según la normativa de la ECE, las actividades inmobiliarias en sí no tienen ninguna excepción; sin embargo, estas actividades a través de 
un fondo inmobiliario podrían incorporarse como una renta por intereses o una renta por dividendos y se les podrían aplicar las reglas relativas al origen de esas rentas pasivas al momento de declararlas, claro está, haciendo las pruebas previas a la incorporación.

Cuando sea la persona jurídica la que incorpore estas rentas en su declaración privada, sí podrá depurarlas mediante la aplicación de los costos de la sociedad.

\section{Dificultades para determinar los obligados a cumplir con la incorporación de las rentas pasivas de la ECE}

Si al realizar el análisis de la ECE se identifica que se cumple con las dos condiciones del Artículo 882, el contribuyente que tenga el 10\% o más deberá cumplir con la obligación de declarar las rentas pasivas a pesar de que no tenga control. Esto nos hace inferir que la ECE deberá informar a sus accionistas o participes cuántos colombianos residentes tienen participación en ella para determinar si hay control, ¿al menos jurídico?, para que el accionista o partícipe que tenga ese $10 \%$ sin control deba incorporarlo en su declaración de renta.

\section{Conclusiones}

La incorporación de las normas de entidades controladas del exterior sin residencia fiscal en Colombia, evidentemente, tuvieron un sustento en las recomendaciones de la OCDE sobre las normas CFC y, seguramente, en la experiencia de otros territorios. Sin embargo, quedan aún muchas oportunidades de mejora para que los contribuyentes logren cumplir con este nuevo requerimiento sin que el esfuerzo sea desproporcionado.
Además, la normativa debe ser más clara para el contribuyente, ya que la forma como la normativa de la ECE remite a otros artículos del Estatuto Tributario puede llevar a los contribuyentes a cometer errores que les pueden acarrear sanciones por inexactitud de las declaraciones.

Cuando se analiza la normativa de países de Latinoamérica como Perú y Chile, se observan muchas semejanzas de incorporación, aunque definitivamente Colombia fue muy radical en la legislación en comparación con estos dos países. Colombia, además, hace caso omiso a recomendaciones relacionadas con los umbrales mínimos, con exenciones y con otros aspectos como los de periodos intermedios; también se observan diferencias específicas en la incorporación de rentas inmobiliarias y las de seguros. En cuanto a la claridad de la redacción de la norma en Colombia, así como en el decreto reglamentario, deja mucho para mejorar, mientras legislaciones como Perú y Chile son más específicas con sus contribuyentes.

Existen otros efectos que, aunque no han sido presentados en esta tesis, es posible que deban ser desarrollados a futuro, como lo enuncia Bravo González, J. (2017, pp. 64-66) cuando hace referencia al impacto de esos ingresos en el IVA, en especial a las diferencias en las declaraciones presentadas ante la administración tributaria o a los requerimientos que haga la UGPP sobre los aportes presuntos que deban realizar los contribuyentes por la información consignada en la declaración de renta de la persona natural y el impuesto de industria y comercio que pueda ser requerido por los rendimientos financieros, por ejemplo, son 
temas expuestos por el autor y que seguramente requerirán de mayor análisis a futuro.

Las normas CFC deben cumplir con su objetivo primordial, que es evitar la erosión de la base gravable, pero preservando la igualdad y la equidad del sistema. Sin embargo, cuando el análisis de las normas de CFC se hace desde la óptica de la persona natural, no se evidencia equidad porque el contribuyente se verá obligado a documentar una serie de situaciones antes de incorporar las rentas de la ECE en su declaración de renta. No tengo la certeza de que los legisladores hayan medido el impacto de la implementación de esta normativa en los contribuyentes y en los asesores tributarios del común, quienes definitivamente deben especializarse para prestar un servicio de mejor calidad a los contribuyentes, tendiente a evitar errores en depuración, liquidación y presentación de sus declaraciones privadas.

Yo me pregunto, si Colombia tiene el espejo retrovisor de más de 50 años de experiencia de la incorporación en otros países de las normas $\mathrm{CFC}$, por qué no tomó esa experiencia e hizo una incorporación más práctica que le permitiera lograr el objetivo sin poner a los contribuyentes a hacer un esfuerzo desproporcionado para cumplir con la reglamentación, así como a sus funcionarios, porque no podemos olvidar que, como lo narra Bravo González, J. (2017, p. 39), el concepto C-776 de 2003 de la Corte Constitucional del M.P. Dr. Cepeda, M. J. "que el principio de eficiencia tributaria no solo se concreta con el logro de un mayor recaudo de tributos con el menor costo de operación posible, sino que también 'se valora como un principio tributario que guía al legislador para conseguir que la imposición acarree el menor costo social para el contribuyente en el cumplimiento de su deber fiscal (gastos para llevar a cabo el pago del tributo)"'. No podemos tampoco desconocer que la administración tributaria carece de funcionarios competentes que orienten efectivamente al contribuyente cuando tiene alguna duda sobre la aplicación de la ley en asuntos nacionales de manejo recurrente, cómo va entonces la Administración de impuestos a apoyar a los contribuyentes en el cumplimiento de esta nueva reglamentación sin tener la experiencia y la capacitación requerida.

Otro aspecto que va a generar dificultades en la aplicación del régimen es para las personas que omitieron la obligación de normalizar sus activos en el exterior y que ahora, como consecuencia de la reforma tributaria, deben incorporar las rentas pasivas de la ECE.

Ahora bien, con referencia a la información que debe entregar el contribuyente a la administración de impuestos, esta solo está contemplada para los declarantes que deban remitir información en medios magnéticos a la DIAN, según la resolución 060 del 30 de octubre de 2017. De acuerdo con esta resolución, dichos contribuyentes deberán incluir en el formato 1036 toda la información que permita identificar la ECE, así como los criterios de vinculación y la forma de control.

Desde el punto de vista de la administración, se esperan comunicados o circulares donde se aclare al contribuyente aquellos aspectos que puedan estar en duda con respecto a la aplicación de la normativa ECE. También sería importante que la administración 
tributaria considerara mejorar sus servicios informáticos y de orientación al contribuyente de forma práctica sobre la aplicación efectiva del régimen, si es que la intención es el recaudo vía incremento de las bases gravables y no recaudos vía sancionatoria, que es lo que termina sucediendo con las reglamentaciones que han sido realmente un fracaso. También será importante conocer de la administración qué documentación probatoria debería tener el contribuyente para dar soporte legal a los cálculos que haga y que presente en sus declaraciones, es decir, qué documentación debe presentar, ya que, como puede observarse, la reglamentación no contempló la documentación probatoria, como sí se hizo en otros países, así como una aclaración sobre los requisitos que se deben cumplir para acreditar los ingresos y gastos relacionados con esas rentas, y qué requisitos deberán tener los documentos que emitan las entidades del exterior para probar los impuestos pagados en el exterior por la ECE. Hacer campañas públicas, así como en su momento se hizo con la normalización tributaria, sería ideal para llevar la tributación a otro nivel.

Deben los legisladores complementar las normas ECE con la cobertura de las rentas por seguros que no fueron incorporadas en la legislación de 2016, y también incorporar la sustancia en la evaluación de las rentas de las ECE, con el fin de determinar si se prestan servicios o se realizan actividades reales. Podría también ser incorporada en la legislación una normativa relativa a que si el impuesto es satisfecho en la jurisdicción donde la ECE tiene su residencia a una tasa superior a la de Colombia, o por lo menos si se satisface un umbral mínimo del $70 \%$ o el $80 \%$ del impuesto pagado en Colombia, la entidad quedaría fuera del régimen de ECE. Esto sería un tamizaje importante para la administración, que le permitiría disminuir su carga administrativa, optimizar la implementación de este nuevo régimen y cumplir realmente el objetivo planteado. Obviamente, las normas ECE deben ser complementarias con normas locales o con la actualización de las vigentes, como es el caso de la fórmula para depurar el crédito fiscal que pueda compensarse por el impuesto satisfecho por la ECE.

\section{Referencias}

Abugattas, N. P. (2014). "Análisis crítico a las nuevas normas CFC introducidas en la Ley 20.780", en Anuario de derecho tributario. 6, pp. 17-61. Recuperado de http://derecho.udp.cl/wp-content/uploads/2016/08/ analisis_critico-1.pdf.

Almudi, C. J. M. (2017). El establecimiento de normas de transparencia fiscal internacional más eficaces frente a la erosión de bases imponibles y el traslado de beneficios (Acción 3). El plan de acción sobre Erosión de Bases Imponibles y Traslado de Beneficios (BEPS): G20, OCDE y Unión Europea, 13.pp.297-330. Thomson Reuters ARANZADI.

Altamirano, A. C. (2005). "Transparencia fiscal internacional. Normas tributarias para la prevención de la elusión internacional", en Publicaciones Instituto Peruano de Desarrollo Tributario, 43. p. 13. Recuperado de http://www.ipdt.org/editor/docs/01_Rev43_ACA. pdf.

Araque.C. J. H.; Araque, D. J. D y otros (2017). Nueva reforma tributaria Ley 1819 de 2016 explicada. pp. 201-206. Grupo Editorial Nueva Legislación SAS.

Araya Z., P. (2016). Cumplimiento tributario de las rentas pasivas (Tesis de maestría). Chile.

Universidad de Chile. Recuperado de http://repositorio.uchile.cl/handle/2250/144268.

Asociación Iberoamericana de Tribunales de Justicia Fiscal o Administrativa (1996). Diccionario Fiscal de la Asociación Iberoamericana de Tribunales de Justicia Fiscal o Administrativa. Recuperado de http://www.aitfa.org/index.php/men-publicaciones/ diccionario. 
Bravo, J. de D. (2018). "Análisis jurídico y aspectos relevantes de la Ley 1819 de 2016", en Jornadas Colombianas de Derecho tributario, Derecho aduanero y Comercio exterior. pp. 19-67. Instituto Colombiano de Derecho Tributario (2018).

Bárcenas, A. (noviembre 2016). Artículo de la Comisión Económica para América Latina y el Caribe. Paraísos fiscales y el financiamiento de la Agenda 2030. Cepal.

BBC Mundo (abril de 2016). ¿Cómo y cuándo se inventaron los paraísos fiscales? Redacción Tomado de: www.bbc.com/mundo/noticias/2016/04/160408_ historia_paraisos_fiscales_offshore_finde.

Cabrera Cabrera, O. S. (2017). El régimen de entidades controladas del exterior (ECE): la Ley 1819 de 2016 frente a la Acción 3 del plan BEPS. El impacto de la Ley 1819 de 2016 y sus desarrollos en el sistema tributario colombiano, Tomo II, Impuesto sobre la renta para personas naturales y tributación internacional. $25 \mathrm{pp}$. 211-244. Pizza, J. R; Castro, J. M. Bogotá: Universidad Externado de Colombia.

Cabrera Cabrera, O. S. (2017). El régimen de entidades controladas del exterior (ECE) de la Ley 1819 de 2016, Análisis crítico de la Reforma Tributaria Ley 1819 de 2016.3 pp. 385-410. Pizza, J. R. Bogotá: Universidad Externado de Colombia.

Castro, J. M. (2017). El gravamen de los dividendos en la Reforma Tributaria de la Ley 1819 de 2016, Análisis crítico de la Reforma Tributaria Ley 1819 de 2016.2,pp. 323-345. Pizza, J. R. Bogotá: Universidad Externado de Colombia.

Código de Comercio. (1971). Diario Oficial No. 33.339 del 16 de junio de 1971. Colombia. Recuperado de http://www.secretariasenado.gov.co/senado/ basedoc/codigo_comercio.html.

Cortés, C. A. (2016). Acción 3: Refuerzo de la normativa sobre CFC. Reporte final de la tercera ( $\left.3^{\mathrm{a}}\right)$ Acción y su impacto en la legislación y práctica colombiana, Resultados del Plan de acción BEPS y su aplicación en Colombia. pp. 57-87. Instituto Colombiano de Derecho Tributario.

Delgado, A. (2017). Modificaciones de fiscalidad internacional en la reforma tributaria de 2016. El impacto de la Ley 1819 de 2016 y sus desarrollos en el sistema tributario colombiano, Tomo II, Impuesto sobre la renta para personas naturales y tributación internacional. 22 pp.133-159. Pizza, J. R; Castro, J. M. Bogotá: Universidad Externado de Colombia.
Delgado, A. (2017). La fiscalidad internacional en la Reforma Tributaria de 2016. Análisis crítico de la Reforma Tributaria Ley 1819 de 2016.3 pp. 377379. Pizza, R.J.R. Bogotá: Universidad Externado de Colombia.

Estatuto Tributario (1995). Artículo 260-1 del Estatuto Tributario, numeral 3 del literal $b$, del ordinal 1 . Colombia. Recuperado de http://estatuto.co/.

Guisado, P.; Sánchez, J. (2016). Jorge Mandes, el artífice del plan para eludir a Hacienda. Recuperado de: www.elmundo.es/deportes/football-leaks/2016/12 /03/5841ecb5468aeb62028b45e2.html.

Jefatura del Estado (2014). "Ley 27/2014, de 27 de noviembre, del Impuesto sobre Sociedades", en Agencia Estatal Boletín Oficial del Estado. 228,96939-97097. España. Recuperado de https://www.boe.es/buscar/ pdf/2014/BOE-A-2014-12328-consolidado.pdf.

Ministerio de Hacienda (1967). Decreto 40: Reglamenta operaciones reajustables de los bancos de fomento. Biblioteca del Congreso Nacional de Chile. Chile. Recuperado de https://www.leychile.cl/Navegar?idN orma=99246\&idVersion=1967-01-20.

Ministerio de Hacienda (2016). Ley núm. 20.899: Simplifica el sistema de tributación a la renta y perfecciona otras disposiciones legales tributarias. Biblioteca del Congreso Nacional de Chile. Chile. Recuperado de https://www.leychile.cl/Navegar?idNorma=10873 42\&idParte=\&idVersion=2017-12-01 .

Monsalve, T. R. (2018). Impuesto 2018, Estatuto Tributario concordado, últimas reformas tributarias decretos reglamentarios del E.T. Centro Interamericano Jurídico Financiero. Recuperado de https:// catalogo.cecar.edu.co/cgi-bin/koha/opac-detail. pl?biblionumber $=29796$.

Ocampo, H. A. (2016). "Propuesta de reglas de transparencia fiscal Internacional para Colombia basado en el derecho comparado y el Plan BEPS de la OCDE", en Revista Instituto Colombiano de Derecho Tributario. 75, 95-142. Recuperado de http://www. icdt.co/publicaciones/revistas/Revista75/Articulo4/ PUB_ICDT_ART_OCAMPOT.HenryAlejandro_PropuestadereglasdeTransparenciaFiscalInternacionalparaColombia_RevistaICDT75_Bogota_16.pdf.

Organización para la Cooperación y el Desarrollo Económicos (OCDE) (2012). “¿Qué es la OCDE?”, en OCDE Publishing. Recuperado de https://www.oecd. org/centrodemexico/publicaciones/ 
Organización para la Cooperación y el Desarrollo Económicos (OCDE) (2014). "Plan de acción contra la erosión de la base imponible y el traslado de beneficios", en $O E C D$ iLibrary. P. 7. Recuperado de http:// www.aedf-ifa.org/FicherosVisiblesWeb/Ficheros/ Fichero79.pdf

Organización para la Cooperación y el Desarrollo Económicos (OCDE) (2016). "Diseñar Normas de Transparencia Internacional Eficaces", en $O E C D i L i$ brary. p. 3 Recuperado de http://www.oecd-ilibrary. org $/$ docserver/download/2315304e.pdf?expires $=1518$ 972995\&id=id\&accname $=$ guest $\&$ checksum $=3$ BAEC 4 7327F9DA2559C43A3C918C72BE

Organización para la Cooperación y el Desarrollo Económicos (OCDE). (2010). Modelo de convenio tributario sobre la renta y sobre el patrimono (versión abreviada)", en $O E C D$ iLibrary. Instituto de Estudios Fiscales. Recuperado de http://www.gerens.cl/gerens/ ModeloConvenioTributario.pdf

Redmiles, M.; Wenrich, J. (2007). "A history of controlled foreign corporations and the foreign tax credit", In Statistics of Income SOI Bulletin, 27 (1), 129-135. Recuperado de https://www.irs.gov/pub/ irs-soi/historycfcftc.pdf

Rojas, N. J. (2013). "Transparencia fiscal internacional: Colombia hacia su tránsito", en Revista impuestos de Colombia, 120, 4-9. Recuperado de http://legal. legis.com.co/document?obra=rimpuestos\&document =rimpuestos_7680752a7e39404ce0430a010151404c
Rozo, G. C. (2017). Régimen de entidades controladas del exterior. Comentarios a la reforma tributaria estructural, Ley 1819 de 2016, pp. 188- 198. Instituto Colombiano de Derecho Tributario.

Superintendencia Nacional de Aduanas y de Administración Tributaria (SUNAT) (2004). Texto único ordenado de la ley del impuesto a la renta Decreto supremo $\mathrm{n} .^{\circ} 179-2004-\mathrm{ef}$. Recuperado de http://www. sunat.gob.pe/legislacion/renta/ley/

Toledo, C.; Ramírez, M. (2017). "La Acción 3 de BEPS, en el contexto de Colombia, Perú y Chile", en Actualidad Jurídica Uría Menéndez. 45, pp. 39-49 Recuperado de www.uria.com/documentos/publicaciones $/ 5316 /$ documento/art04.pdf?id=6966

Velasco, J. D.; Fernández, P. (2018). “Análisis crítico a las presunciones de control y sujeción al régimen de Entidades Controladas del Exterior en Colombia", en Jornadas Colombianas de Derecho tributario, Derecho aduanero y Comercio exterior. pp. 385-390. Instituto Colombiano de Derecho Tributario (2018).

Villagra, R.A. (2013). “Análisis crítico del régimen de transparencia fiscal internacional vigente en el Perú a partir del 2013", en Themis: Revista de Derecho. 64, p. 54. Perú. Breve historia de la transparencia tributaria, de Alberto Barreix, Jerónimo Roca y Fernando Velayos. Junio de 2016. Del BDI (Banco Interamericano de desarrollo), http:/www.publications.iadb.org/

Zarama, F.; Zarama, C. (2017). Reforma tributaria comentada Ley 1819 de 2016. pp. 377-394. Bogotá: Legis, Información y soluciones. 